\title{
Functional Anatomy: A Taxonomic Proposal
}

\author{
Ingvar Johansson, Barry Smith, Katherine Munn, Nikoloz Tsikolia, \\ Kathleen Elsner, Dominikus Ernst, and Dirk Siebert \\ Institute for Formal Ontology and Medical Information Science (IFOMIS), \\ University of Saarland, Saarbrücken, Postfach 151150, D-66041, Germany
}

\begin{abstract}
It is argued that medical science requires a classificatory system that (a) puts functions in the taxonomic center and (b) does justice ontologically to the difference between the processes which are the realizations of functions and the objects which are their bearers. We propose formulae for constructing such a system and describe some of its benefits. The arguments are general enough to be of interest to all the life sciences.
\end{abstract}

\section{Traditional Anatomy and Functional Anatomy}

The idea to be outlined in this paper is that there is an as yet unexplored taxonomical approach within the life sciences, in particular within medicine, which we call pure functional anatomy. Pure functional anatomy is complementary to the purely structural approach to anatomy exemplified by the Foundational Model of Anatomy (Cornelius and Mejino, 2003), while at the same time remaining distinct from pure physiology. Physicians as well as medical scientists are of course already working with a large amount of more or less implicit knowledge of functional anatomy. Our proposal is that this knowledge be made explicit and that it be carefully systematized in the form of a new kind of taxonomy.

On one traditional conception, anatomy describes only the structures of the body, leaving functions and processes to physiology. The aim of anatomy, on this conception, is to divide the human body into its spatial parts by reference to the physico-chemical composition and spatial arrangement of these parts, including topological relations such as adjacency and connectedness. Even to the very earliest anatomists, it must have been apparent that the various organs in the body such as the lungs, heart, and kidneys are composed of different kinds of material. And it was easy to view the skeletal system as a spatial coordinate system in relation to which all other anatomical entities could be assigned their places.

Philosophers often say "no entity without identity." But one can equally, for entities in space and time, say that there is "no entity without boundary." What, then, determines the boundaries of anatomical entities? For many of them, for example the separate bones of the foot, anatomists select a pre-existing discontinuity in the physico-chemical composition of reality - between the material entity on the one hand and its surroundings (including a liquid medium) on the other. But when it 
comes to parts of entities made of more homogeneous material, the boundaries must of course be determined in some other way. They have to be in some sense a product of our demarcation as, for example, they are when speak of the upper and the lower portions of the femur. In other words, some boundaries in the body are bona fide and some are fiat (Smith, 2001). Similarly, when a part of the body recognized by anatomists is made up of non-connected parts (e.g., the endocrine system) then here, too, the circumcluding boundary must be a product of our demarcation. Many anatomical parts such as the skull, the ribs, the chest bone, and the pelvis have a boundary that is partly bona fide and partly fiat. Often, such mixed boundaries are constituted by a large bona fide portion complemented by a small fiat boundary drawn for example at the point where organs are connected via conduits for the passage of substances in or out.

But there is also another way to determine spatial boundaries. One can select boundaries in such a way that the entity delineated is a function bearer, that is, a unity which as a whole performs a certain function. The musculoskeletal system is not a unity from a structural point of view, but from a functional point of view it is. The muscles and skeleton are grouped together because their unity is what bears the function: to move the body.

Of course, many functions are such that their bearers are single anatomical parts already structurally discerned (the heart has the function to pump blood, the liver has the function to produce glycogen, and so on). Our point is that this is not the case for all functions. If we are correct, then it follows that there are two differently demarcated kinds of anatomical parts in the body: spatial-structural parts and spatialfunctional parts. Each can ground an anatomy of its own, notwithstanding the fact that some parts are simultaneously both spatial-structural and spatial-functional. We will call the results of such demarcation pure structural anatomy and pure functional anatomy, respectively. These anatomies are both partonomies and taxonomies, i.e., the spatial parts discerned are also given either a structural or a functional description.

Many anatomical textbooks, old as well as new, can be conceived as being neither purely structural nor purely functional, for they contain demarcations with mixed motivations. When, for instance, the heart muscle is regarded as a part of the circulatory system rather than of the muscle system, this seems not to reflect an essential structural difference between this muscle and the other muscles.

The term "functional anatomy" is widely used. As this paper is being finalized (November, 2004), Google ${ }^{\mathrm{TM}}$ lists 119.000 entries for this term. There are papers about the functional anatomy of limbs, of the knee-joint, of the hypothalamus, and many more. Thus far, however, there seems to exist no clear statement of the principles governing functional anatomy, indeed of what the word 'functional,' and of cognate words such as 'regulation,' 'inhibition,' and 'promotion' might mean. We thus believe that the construction of a purely functional anatomy can be of more than merely taxonomic interest. By helping to throw light on the meaning of 'function' it can have implications for our understanding of a range of important contemporary developments in the life sciences, for example as are clustered around the enterprise of 'functional genomics.' It can contribute also - and no less importantly - to our understanding of clinical medicine (which is focused at least in part on the alleviation of malfunctions). Even medical technology might benefit, since artificial body parts are meant to preserve old functions with the help of new structures. 


\section{Constituent Functions}

Function talk seems to come naturally to people who know a lot about the human body and its parts. Many philosophers of science claim nevertheless that such talk should be reduced to what they conceive as 'teleology-free' talk of causality or natural selection (see section 6.2 below). Their reasoning turns on the fact that functions would be features of objects directed in some sense towards even a distant future, and such features make no sense in a scientific world view. They reflect, rather, an anthropomorphic imputation. We think, however, that the proposed reduction of function talk is not feasible, and that there is a non-reducible notion of function, which we will call constituent function, that reflects an objective feature of the world. The notion we have in mind might also have been expressed by means of terms such as 'component function' or 'part-to-whole function.' What all these terms have in common is that they help us keep in mind that constituent functions are in every case relational in character (Johansson, 2006).

As point of departure for our taxonomic proposal, we have taken the human body regarded as a functional unit whose short-term function we can think of provisionally as: to preserve the life of the organism. Relative to this function of life-preservation most bodily functions are constituent functions, i.e., they are functions in virtue of their relation to this larger whole. What we mean when we say that the function of the heart is to pump blood is that the heart, when inside a living body, both acts and has a disposition to act in a certain way to contribute to the realization of this larger function on the part of that whole organism which is its host.

It follows from this account that an entity does not have a constituent function in and of itself in the way in which, for example, mass is assumed to inhere in Newtonian corpuscles or human conscious intentions are assumed to inhere in persons. Functions that are objective monadic properties in this sense, we call intrinsic functions. It is functions of this sort which have been the targets of standard attempts at reduction. We here leave open the question whether such intrinsic functions exist, noting only that it is at least in principle possible for an entity to be the bearer of both intrinsic and constituent functions.

In light of these remarks we can now advance a first revision of the provisional account of the function of the whole (living) human body given above. For we now see that the latter can be regarded either:

(a) as a constituent function of a functional unit that is larger than the human body;

(b) as an intrinsic function of the human body; or

(c) as some sort of a projection, i.e., as a feature that is merely imputed to the human body by perceiving and language-using human beings, but which corresponds to no underlying feature of reality.

None of these options is without its corresponding problems. If, as according to (a), the human body has its function of preserving life only if it is itself part of some larger functional whole, then what is to be said about that whole and its function? An infinite regress arises if one allows for constituent functions only. Therefore, from a philosophical point of view, at some stage we have to bring the chain of ever larger circumcluding wholes to an end, and then we face a choice between options (b) and (c). 
If we choose (b), we are confronted with the problem of how to make intrinsic functions consistent with natural science. If the human organism in and of itself has at a certain point in time the function to preserve its life for some time to come, then the organism as a causal-energetic whole must somehow be able to be directed towards these as yet non-existing points of time. Ordinary vectors such as velocity and acceleration are not like this. They are directed only toward the very next instant of time, not toward anything temporally more distant. Option (b) therefore seems to make functions too similar to conscious human intentions to be parts of natural science.

Third, if we opt for (c), as for instance John Searle (1995) and Mohan Matthen (1997) do, we seem to license some sort of cultural relativity or even personal subjectivity to enter into science.

Quite a trilemma. How to choose between (a) an infinite regress, (b) an anthropomorphizing of nature, and (c) subjectivity? Our solution is simple, since it is of the cutting-the-Gordian-knot variety. We claim that, independently of whether or not the self-preservation of the whole human body is itself either a constituent function, an intrinsic property of the body, or only a projected feature, still all of the constituent functions of the body are objective features of the world. This move suffices to make functional anatomy a tractable scientific enterprise.

Perhaps an analogy can give further credence to our view that constituent functions are objective. As an action can be objectively rational in its relation (as a means) to a pre-given end - even if this end is itself completely irrational - so a spatial part can be objectively functional in its relation to an encompassing pre-given functional whole even if the corresponding function of the whole is not an objective intrinsic function but rather a mere subjective projection (Johansson, 2006).

\section{The Proposal Visualized}

We grant that a functional unit may have more than one function. For instance, for the stomach we can distinguish at least: Function 1: to break down the chyme and Function 2: to move the broken-down chyme into the duodenum. Typical functions of other parts are: to store (something), to pump, to protect, to produce, to open, to close, to absorb, and to expel. The terminology of Function 1, Function 2, etc., as we use it in what follows, does not imply any ranking of functions in order of importance. It is designed merely to provide a reminder of the complex array of different kinds of dependence, support and regulation relations which can be detected in the different constituent functions of a complex organism like the human being.

We can distinguish a first level of spatial-functional parts of the human body as in the figure below. 
The Human Body (Function 1: to preserve the life of the organism):

\begin{tabular}{|c|c|c|}
\hline $\begin{array}{c}\text { Circulatory } \\
\text { system }\end{array}$ & $\begin{array}{c}\text { Alimentary } \\
\text { system }\end{array}$ & $\begin{array}{c}\text { Respiratory } \\
\text { system }\end{array}$ \\
\hline $\begin{array}{c}\text { Senso-motoric } \\
\text { system }\end{array}$ & $\begin{array}{c}\text { Excretory } \\
\text { system }\end{array}$ & $\begin{array}{c}\text { Immune } \\
\text { system }\end{array}$ \\
\hline $\begin{array}{c}\text { Musculoskeletal } \\
\text { system }\end{array}$ & $\begin{array}{c}\text { Vegetative regulation } \\
\text { system }\end{array}$ & $\begin{array}{c}\text { Integumentary } \\
\text { system }\end{array}$ \\
\hline
\end{tabular}

Figure 1. Spatial-functional parts of the human body

Figure 1 makes visible those parts of the body to which constituent functions can be assigned relative to the function of the human body as a whole. Each of these parts makes a necessary contribution to the short-term preservation of the organism's life (Smith et al., 2004). It is, as it were, a window on reality. But as we shall see, it is a window of a special sort, which can give way to nine other windows that offer finer grained views on their respective part of the same reality via what we can think of as a process of ontological zooming (Bittner and Smith, 2001). Figure 2 provides the result of zooming in on the circulatory system:

The Circulatory System (Function 1: to transport substances between bodily systems):

\begin{tabular}{|c|c|c|}
\hline $\begin{array}{c}\text { Circulatory } \\
\text { fluids }\end{array}$ & $\begin{array}{c}\text { Vessel } \\
\text { system }\end{array}$ & Heart \\
& & \\
\hline
\end{tabular}

Figure 2. Spatial-functional parts of the circulatory system

We have identified only one main function of the circulatory system. However, we think that the alimentary system has at least two functions: Function 1: to absorb nutrients from food and Function 2: to expel non-nutrients that come from food.

Zooming in first on the vessel system (Figure 3) and then on the heart (Figure 4) reveals: 
The Vessel System (Function 1: to direct fluids in the body):

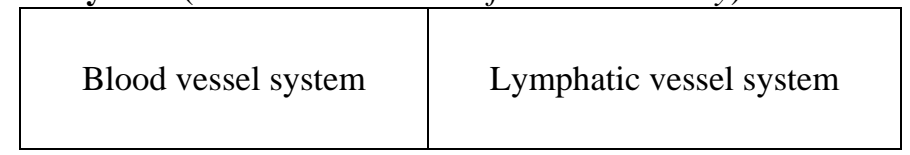

Figure 3. Spatial-functional parts of the vessel system

The Heart (Function 1: to pump blood within the blood-vessel system):

\begin{tabular}{|c|c|c|}
\hline Atria & Ventricles & Valves \\
\hline
\end{tabular}

Figure 4. Spatial-functional parts of the heart

By continuing to zoom in on ever smaller parts, one can zoom down, through the cell and its major spatial-functional parts, down to (functional) biological macromolecules. In this way we can arrive at branches through a tree of successively smaller spatial-functional parts such as the following:

(1) human body $\rightarrow$ circulatory system $\rightarrow$ vessel system $\rightarrow$ blood vessel system

$\rightarrow$ arterial system $\rightarrow$ macrovasculature $\rightarrow$ tunica intima $\rightarrow$ endothelial cells;

(2) human body $\rightarrow$ alimentary system $\rightarrow$ stomach $\rightarrow$ tunica mucosa $\rightarrow$ surface epithelium $\rightarrow$ endocrine cells.

Both the number of spatial-functional parts of a single functional entity within the locus of the human body, and also the number of levels of such parts between the human body as a whole and its macromolecules, seem to be a matter of contingent fact. Thus there seems to be no general laws that predetermine how many spatialfunctional parts an organism has in total.

The human body and all its spatial-functional parts - its function-bearers at successive layers - retain their identity through time. In philosophical jargon, they endure. This means that they are contrasted ontologically with their respective lives, or histories, which do not endure but rather unfold themselves through time in successive temporal parts or phases. The same opposition exists also on the side of the corresponding functions in themselves. Each person's circulatory system has at any moment in time the function to transport substances between bodily systems. The heart at its center has at any moment in time the function to pump blood within the blood-vessel system. Such functions, too, are endurants, and are to be contrasted with their functionings. The latter are processes, subject to a division into temporal parts, as is illustrated for example by the cyclical functional activity of the heart, where each cycle contains two bona fide parts: a diastolic phase and a systolic phase. We regard the distinction between functions and functionings (Johansson, 2004), with their radically distinct ways of existing in time, as an important element of our taxonomic proposal. 
The kinds of temporal parts comprehended by any given functional activity can now be added to the relevant figure. In the case of the heart, this would yield a diagram as follows:

The Heart (Functioning of Function 1):

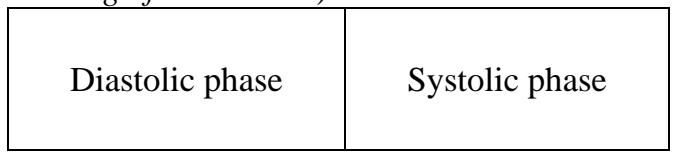

Figure 5. Temporal parts of the functioning of the heart

Of course, many bodily processes, unlike the functional activity of the heart, feature actual rest phases (see end of section 4).

As in the case of our zooming in on spatial-functional parts, so also here: a zooming in on e.g. the diastolic phase of the heart's functioning will reveal smaller temporal parts of this phase.

\section{The Proposal as a Partonomic-Taxonomic Formula}

We now introduce the idea of a taxonomic formula. All the classical biological classifications of sexually reproducing species, which extend downwards from kingdom via phylum/division, class, order, family, and genus to species, might be said to be constructed according to the taxonomic formula: "Define species by means of interbreeding, then order all the species by means of relations of similarity and dissimilarity into a hierarchy of taxa.” A similar taxonomic formula for Mendeleev's original periodic table might read: "The physical and chemical properties of the elements are in periodic co-variation with increasing atomic weights; order the elements on this basis into rows and columns.”

We shall see that the presentation in section 3 is based both on a partonomictaxonomic formula concerned with spatial-functional parts and their functions and on a complementary formula concerned with the temporal parts of the functionings of these functions.

The first and basic formula focuses on spatial-functional parts as they exist in prototypical human organisms at an arbitrary point in time. It might therefore be called a SNAP (for 'snapshot') taxonomic formula (or SNAP formula, for short). Since the second formula takes account of temporal extension, it may be called a timeSPAN taxonomic formula (or SPAN formula, for short) (Grenon and Smith, 2004). The formulae read as follows: 


\section{SNAP}

(a) In the functional unit $\mathrm{A}$,

(b) one function of the spatial part and functional subunit B of $\mathrm{A}$ is:

(c) to $\mathrm{F}$ in relation to $\mathrm{X}, \mathrm{Y}, \mathrm{Z}$, etc.

\section{SPAN}

(d) This function (F) has as temporal parts of its functioning

(e) the phases $P_{1}$ to $P_{n}$.

Since we are talking about a prototypical organism (see also 5.3 below) we do not need, here, to take care of parts that take on some function or other as it were accidentally or as a result of special circumstances.

Our SNAP formula can be used both iteratively and recursively. That is, it can be applied iteratively to all the different parts of a functional unit that belong to the same level, and it can be repeated recursively in the sense that - in keeping with our examples of zooming, above - a subunit on level (b) in the SNAP formula is in the next cycle turned into a unit on level (a), and so forth. Examples are:

(a) In the human body (A),

(b) one function of the circulatory system (B) is:

(c) to transport substances (F) between the bodily systems (X, Y, Z, etc.).

(d) This function (F) has as temporal parts of its functioning

(e) either fiat parts of the continuous fluid flow or bona fide parts demarcated through the substances transported.

And:

(a) In the circulatory system (B),

(b) one function of the heart (C) is:

(c) to pump blood (F) within the blood vessel system (X).

(d) This function (F) has in its functioning as temporal parts

(e) cycles, divided into the diastolic $\left(\mathrm{P}_{1}\right)$ and the systolic $\left(\mathrm{P}_{2}\right)$.

Lines (a) and (b) reflect the requirement that nothing can be a constituent function if it is not the function of some part of a larger functional unit. (Intrinsic functions, whether they exist or not, do not fit our first formula.) A recursive use of the SNAP formula does not imply that the initial unit A disappears; it means only that A drops out of the foreground. For B remains a subunit of A; similarly, $\mathrm{C}$ remains a subunit of $\mathrm{B}$; and so on.

Our formulae allow that some of the partonomic paths between the main organ systems and macromolecules will run through more levels of spatial-functional parts than others. In the general SNAP formula, line (c) says that the function of a certain entity is: to $\mathrm{F}$ in relation to $\mathrm{X}, \mathrm{Y}, \mathrm{Z}$. Here, $\mathrm{F}$ is a variable for functions (depicted by verb phrases - to pump blood, and so on), and the variables $\mathrm{X}, \mathrm{Y}$, and $\mathrm{Z}$ range over entities on every possible level along any given partonomic path. No level constraints are mentioned in clause (c). But if such constraints are found, the formula can easily be adjusted in this respect. 
It should also be noted that the formulae completely disregard the issues which arise when an organ ceases to be a constituent of the relevant larger whole. We might say both that hearts which, during heart transplantations, are in a solution outside any bodily host and hearts which, during open-heart surgeries, are replaced by a heartlung machine have their function only potentially. But we can ignore such cases since the formulae apply only to functions of entities that are in fact parts of larger functional units. Surgery apart, in a living body the heart always has its function actually. This would hold even if, counterfactually, its functioning, like the functionings of a number of glands, contained various kinds of passive phases such as rest phases, waiting phases, guarding phases, and so on. It is one thing for a part or system to have a function only potentially and therefore not to be functioning for this reason, and it is something quite different for it to have a function actually and to be functioning, but only in the sense that, so to speak, it is on duty without needing to do anything.

At any given time and for any given entity $\mathrm{E}$ and kind of function $\mathrm{F}$, one of the following four possibilities must obtain:

(i) E neither actually nor potentially has the function $\mathrm{F}$.

(ii) E has the function F only potentially.

(iii) $\mathrm{E}$ has the function $\mathrm{F}$ actually, but $\mathrm{E}$ is in a passive phase in relation to $\mathrm{F}$.

(iv) $\mathrm{E}$ has the function $\mathrm{F}$ actually, and $\mathrm{E}$ is an active phase in relation to $\mathrm{F}$.

When $\mathrm{E}$ is in a passive phase, it still has the disposition to act in the way required in the active phase. When $\mathrm{E}$ only potentially has the $\mathrm{F}$, it may not have such a disposition.

\section{Some Features of the Formulae}

Some more words about the proposed SNAP-SPAN formulae are necessary in order to make some of their features clear.

\subsection{Sharing spatial-functional parts}

When, in non-evolutionary taxonomies of species, one goes downward from level A to level B to level $\mathrm{C}$, and so forth, it is usually required that no classificatory "diamonds" (Figure 6) should arise.

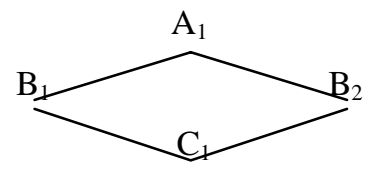

Figure 6. Classificatory diamond 
Our SNAP formula, on the other hand, gives rise to diamond structures. This is due to the fact that it is a partonomic-taxonomic formula, and partonomies may well contain diamonds. Several parts of the human body are spatial-functional parts of two or more larger functional units. For instance, for the oropharynx it holds true that:

(a) In the alimentary system,

(b) function 1 of the oropharynx is:

(c) to make possible food transport from the oral cavity to the esophagus. (a) In the respiratory system,

(b) function 1 of the oropharynx is:

(c) to make possible gas transport between the nasal-oral cavities and the larynx. (a) In the oropharynx,

(b) function 1 of the tunica muscularis is:

(c) to move food into the esophagus. (a) In the oropharynx,

(b) function 1 of the pharyngeal cavity is:

(c) to direct the flow of air.

Figure 7. Partonomic-taxonomic loci for the oropharynx

This means that we can construct the following diamond, where each line (taken in an upward direction) means "is a spatial-functional part of”:

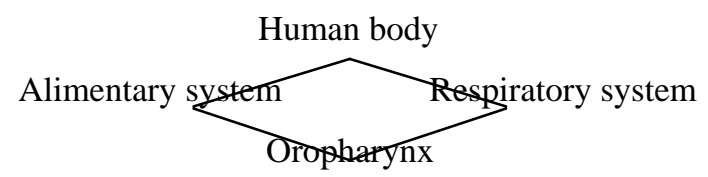

Figure 7. Sharing of spatial-functional part (oropharynx)

Another instance of this kind of part-sharing is afforded by the liver, which belongs to both the alimentary system and the immune system, and almost certainly to other systems as well.

Part-sharing allows for the easy integration of discoveries of new joint functions of already known spatial-functional parts.

\subsection{Multi-Functionality Proper}

It is sometimes taken for granted that nature has worked in such a way that one spatial-functional part can have only one function in relation to one larger functional 
unit. Our taxonomic formulae make no such presumption. We do not say "the function of B in A is," but rather "one function of B in A is." Even within one and the same functional unit a specific spatial-functional part may have more than one function. For instance:

In the alimentary system, function 1 of the liver is: to produce glycogen.
In the alimentary system, function 2 of the liver is: to produce bile.

\subsection{Prototypicality}

It is here taken for granted that an entity that has a constituent function can be functioning better or worse, but that those functionings named in our formulae correspond to the well-functioning cases. Thus the latter might be called prototypical. This does not mean that we think that degrees of functioning can be plotted along some single dimension (indeed, we think that this is impossible). Nonetheless, one may speak in a loose sense of a "distance" between a given token example of functioning and the corresponding prototypical functioning. One kind of zero point is reached when an entity not only functions badly but starts to malfunction; an absolute zero point is of course reached when the entity in question can no longer function at all.

We do not regard the functions designated under the heading 'Function $\mathrm{X}$ ' as being the only possible prototypical functions. A certain functioning may well be related to a number of different prototypes.

In the philosophy of the natural sciences, if not in the natural sciences themselves, concepts involving the feature of prototypicality have often been regarded as scientifically "immature." Our proposal, however, is based on the view that this is wrong (Johansson, 2004). Our functional anatomy is meant to be a canonical anatomy in the sense of Rosse and Mejino (2003). It deals with the general structure of the normal or idealized human body and is to be distinguished from a range of specialized disciplines which deal with non-normal cases and individual organisms.

\section{Background Ideas in the Life Sciences and in Philosophy}

The taxonomic SNAP-SPAN formula we have presented has grown out of general ideas that have been debated for some time in both the life sciences and philosophy. Let us present some of them. 


\subsection{Three-Level Granularity}

All of the life sciences contain, explicitly or implicitly, ideas about hierarchical systems, about levels of functionality, and about granularity. Our proposal comes close to the idea defended by researchers such as Stanley Salthe (1985, pp. 75ff) and D.W. McShea and E.P. Venit (2001, p. 271), who have argued that three such levels should be taken into account. This is because a constituent function $(F)$ is on our view related always to at least three entities. The smallest possible taxonomic unit in functional anatomy, we repeat, has the structure:

(a) In the functional unit $\mathrm{A}$,

(b) one function of the spatial part and functional subunit $\mathrm{B}$ of $\mathrm{A}$ is:

(c) to $\mathrm{F}$ in relation to $\mathrm{X}, \mathrm{Y}, \mathrm{Z}$, etc.

Here, what is designated by $\mathrm{F}$ is related to its bearer (B), a functional whole (A), and at least one entity in relation to which it causes something to happen (X). B, here, designates in every case an entity that belongs to a lower level of granularity than those designated by A, but we have not required that, similarly, entities designated by $\mathrm{X}, \mathrm{Y}$, and $\mathrm{Z}$ belong to an adjacent level that is still lower. However, when this is in fact the case, then our formula conforms to the three-level requirement argued for by Salthe, McShea, and Venit.

\subsection{Scientific Non-Darwinian Functions}

The general suspicion that teleological notions should not be used in the natural sciences emerged from the rise of modern physics; Descartes is only the most famous protagonist for this view. Later, the Darwinian revolution enabled biologists to replace teleological notions such as design of species by the more scientifically defensible natural selection of species. Nonetheless, biologists and physicians have continued to speak of functions - which has made a number of twentieth-century philosophers of science uncomfortable. Their solution has been to claim that such modern function talk is innocent, because it can always be translated into equivalent, but more cumbersome, talk about causes, dispositions, adaptations, and so on, though they have argued among themselves about what the exact translation should look like. Classical "etiological analyses" include those of Larry Wright (1973) and Ruth Millikan (1989). (For overviews see Peter Melander (1997) and Mark Perlman (2004). For criticisms and alternatives see Christopher Boorse $(1976,2002)$ and Robert Cummins (1975, 2002); for more good criticism, see also Richard Manning (1997).)

Such etiological approaches presuppose that all function talk is explanatory talk; so that the function of A is referred to only in order to explain why A exists. Our attempt to defend a natural-scientific concept of function under the heading of "constituent function" rejects this presupposition. In conformity with the views of Cummins and Boorse, we insist that much function talk is either purely descriptive or explains how something works when it exists.

As already indicated, our view comes close to Cummins's so-called "intrasystemic role analysis" (Melander 1997, p. 51) and to Boorse's "general goal-contribution analysis" of functions. Cummins claims: "To ascribe a function to something is to 
ascribe a capacity to it which is singled out by its role in an analysis of some capacity of a containing system" (1994, p. 67; our italics); and Boorse analyzes "functions as causal contributions to goals of a goal-directed system" (2002, p. 63). Both regard functions (as we do) as relational entities, but they differ from each other and from us in the way they look upon the whole of which a function is a part. Cummins puts no restrictions at all on what a "capacity of a containing system" might be, whereas Boorse requires that the whole be goal-directed. Boorse treats the concept of goaldirectedness as a primitive concept, and he "takes goal-directedness to be an objective, non-mental property of all living organisms” (2002, p. 63-64).

In what way, then, does our approach differ from those of Cummins and Boorse? Mainly in these respects:

(i) Cummins is a semantic reductionist, who tries to do away with the concept of function - though he still employs the concept of "role," which is itself, in our eyes, a kind of function concept.

(ii) Boorse makes his analysis depend on the existence of objective nonmental goals, i.e., on what we have called "intrinsic functions."

(iii) Neither Cummins nor Boorse has noted the trilemma and objectivity problems mentioned in section 2, and have therefore not explained how their analyses differ from merely subjective ascriptions of roles and goals.

Arno Wouters has claimed that it can be doubted whether philosophers' "function concept has anything to do with function talk in biology and psychology," and that philosophers "may easily end up with a theory that is philosophically well-founded but gives us no insight whatsoever into the practice of inquiry” (2003, p. 233). We hope that such an accusation cannot be made with regard to the concept of constituent function advanced above.

\subsection{Bipartite Taxonomy}

We have put forward two formulae, one for functions and one for functionings. This idea relates to discussions between philosophers and information scientists about socalled "top-level ontologies" within information science; a top-level ontology contains a classification of the most general types of entities and relations in reality. From the point of view of a realist philosophy, however, most such ontologies do not do adequate justice to the fundamental difference between enduring entities and processes. Enduring entities exist as wholes in a single instant of time in a way in which processes cannot. Enduring entities can exist from one moment to the next; processes unfold in successive phases (they can be segmented into parts along the temporal dimension). Both enduring entities and processes belong to one and the same reality, our world; but they might nonetheless be said to constitute two different, albeit related, ontologies, which have been termed SNAP and SPAN, respectively (Grenon and Smith, 2004). In line with this duality, our taxonomic proposal presents functions (as enduring entities) as part of the SNAP-ontology, and functionings (as processes) as part of the SPAN-ontology. Conversely, the structure of our proposal supports the view that the difference between SNAP-entities and SPAN-entities is far more radical than any difference within either of these ontologies. In our proposal 
only the SNAP formula features a hierarchical nesting of its own, and one might even say that the complementary SPAN formula is parasitic upon the SNAP formula. But both formulae are needed for an accurate representation of the functional anatomy of the organism.

\section{Concluding Remarks: Possible Modifications of the Formulae}

It is easily seen that the proposed taxonomic formulae allows for various kinds of modifications.

\subsection{Substitute Functions}

It is well known that when certain bodily organs are injured in such a way that they are no longer capable of performing their function, then some other spatial-functional part will take over. After a splenectomy, for instance, the liver takes over some of the spleen's functions. In order to integrate such data, the original formulae can, and must, be amended with the addition of the concept of substitute functions and functionings (and of related concepts of plasticity and redundancy).

\subsection{Information Transmission and Regulation}

Our formulae have been worked out with the classical bodily systems in mind and using functions such as: to transport (something), to direct, and to pump. For the time being, the transmission of information and regulation, such as signal transduction and cell-to-cell communication, are beyond our focus. In principle, however, our formulae can be extended to allow for functions and functionings of these sorts also. Probably, such an extension is necessary in order to take care of all spatial-functional parts of the immune system.

\subsection{Disease Taxonomies}

As we said in section 5.3, every actually existing constituent function's functioning can be more or less prototypical, and it can even be a case of malfunction. Now, given our defense of the concept of function, it seems to be true to say that somatic diseases consist either in a malfunctioning of or in a complete loss of at least one of the body's constituent functions. Since every possible taxonomic formula for malfunctionings and every possible formula for loss of functions refers to a corresponding constituent function, all diseases can be related to the formulae we have put forward above. The disease taxonomy that is implied by this insight might thus also bring new order into the multifarious world of diseases. 


\section{References:}

Bittner, T. and B. Smith (2001). Vagueness and Granular Partitions. In: Proceedings of the Conference on Formal Ontology in Information Systems - FOIS 2001, pp. 309-320. ACM Press, New York.

Boorse, C. (1976), Wright on Functions. Philosophical Review 85: 70-86.

Boorse, C. (2002), A Rebuttal on Functions. In: A. Ariew et al. (eds.), Functions. New Essays in the Philosophy of Psychology and Biology, pp. 63-112. Oxford University Press, Oxford.

Cummins, R. (1975). Functional Analysis. In: E. Sober (ed.), Conceptual Issues in Evolutionary Biology, pp. 49-69. MIT Press, Cambridge. Mass., second ed. 1994; originally in Journal of Philosophy 72: 741-765.

Cummins, R. (2002). Neo-Teleology. In: A. Ariew et al. (eds.), Functions. New Essays in the Philosophy of Psychology and Biology, pp. 157-172. Oxford University Press, Oxford.

Grenon, P. and B. Smith (2004). SNAP and SPAN: Towards Dynamic Spatial Ontology. Spatial Cognition and Computation 4: 69-103.

Johansson, I. (2004). Functions, Function Concepts, and Scales. The Monist 86: 96-115.

Johansson, I. (forthcoming 2006). The Constituent Function Analysis of Functions. In: H.J. Koskinen, S. Pihlström and R. Vilkko (eds.), Science - A Challenge to Philosophy. Papers presented at the XV Internordic Philosophical Symposium, Helsinki, May 2004. (Also at: http://hem.passagen.se/ijohansson/index.html.)

Manning, R.N. (1997). Biological Function, Selection, and Reduction. British Journal for the Philosophy of Science 48: 69-82.

Matthen, M. (1997). Teleology and the Product Analogy. Australasian Journal of Philosophy 75: 21-37.

McShea, D.W. and E.P. Venit (2001): What Is a Part? In: Wagner and Wagner (eds.), The Character Concept in Evolutionary Biology, pp. 259-284. Academic Press, London,

Melander, P. (1997). Analyzing Functions. An Essay on a Fundamental Notion in Biology. Almkvist \& Wiksell International, Stockholm.

Millikan, R.G. (1989). In Defence of Proper Functions. Philosophy of Science 56: 288-302. 
Perlman, M. (2004), The Modern Philosophical Resurrection of Teleology. The Monist 86: 3-51.

Rosse, C. and J.L. Mejino Jr. (2003). A reference ontology for biomedical informatics: the Foundational Model of Anatomy. Journal of Biomedical Informatics 36: 478-500.

Salthe, S.S. (1985). Evolving Hierarchical Systems. Their Structure and Representation. Columbia University Press, New York.

Searle, J. (1995), The Construction of Social Reality. The Free Press, New York.

Smith, B. (2001). Fiat Objects. Topoi 20: 131-148.

Smith, B., K. Munn, and I. Papakin (2004). Bodily Systems and the SpatialFunctional Structure of the Human Body. In: D. Pisanelli and M. Domenico (eds.), Ontologies in Medicine: Proceedings of the Workshop on Medical Ontologies, Rome, October 2003, pp. 39-63. IOS Press, Amsterdam.

Wouters, A. (2003). Essay Review: Philosophers on Function. Acta Biotheoretica 51: 223-235.

Wright, L. (1973). Functions. In: E. Sober (ed.), Conceptual Issues in Evolutionary Biology, pp. 27-47. MIT Press, Cambridge. Mass., 1994; originally in Philosophical Review 82: 139-168.

\section{Acknowledgements:}

For comments on earlier versions of this paper we would like to thank Pierre Grenon, Bert Klagges, Anand Kumar, Igor Papakin, and two anonymous referees. The paper was written under the auspices of the Wolfgang Paul Program of the Alexander von Humboldt Foundation, the European Union Network of Excellence on Medical Informatics and Semantic Data Mining, and the Volkswagen Foundation under the auspices of the project "Forms of Life." 